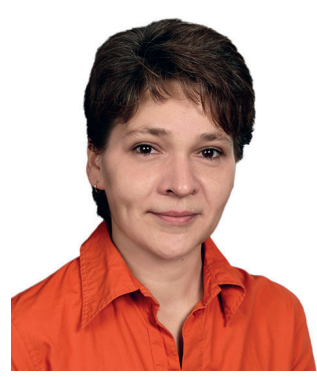

\title{
Chronisch-entzündliche Hauterkrankungen und psychische Erkrankungen - Zusammenhänge beachten und daran denken
}

Sandra Philipp

Hautarztpraxis Dr. M. Friedrich/Dr. S. Philipp, Oranienburg, Deutschland

Abstract aus Farzanfar D, Dowlati Y, French LE, Lowes MA, Alavi A: Inflammation: a contributor to depressive comorbidity in inflammatory skin disease. Skin Pharmacol Physiol 2018; 31: 246-251.

\author{
Keywords \\ Inflammation - Depression - Skin disorders - Psoriasis - Atopic \\ dermatitis · Hidradenitis suppurativa
}

\begin{abstract}
The prevalence of affective disorders such as depression and anxiety is particularly high in patients with autoimmune diseases, including inflammatory skin diseases such as psoriasis, atopic dermatitis, and hidradenitis suppurativa. A dysregulated immune response has been linked to the precipitation of depression in many patient populations. However, studies examining the extent to
\end{abstract}

which the underlying skin disease inflammatory processes contribute to depression and a subsequent decline in quality of life are limited. The published literature over the past 5 years was reviewed for evidence of a relationship between depression and inflammatory processes in the context of skin pathology. The findings, particularly the evidence from interventional clinical trials of targeted anti-cytokine therapies, suggest that proinflammatory cytokines associated with several skin diseases may be causally linked with the coexistent depressive symptomology.

(c) 2018 S.Karger AG, Basel

\section{KARGER}

(C) 2019 S. Karger GmbH, Freiburg 


\section{Transfer in die Praxis}

\section{Hintergrund}

Psoriasis (Ps), Hidradenitis suppurativa (Hs) und atopische Dermatitis (AD) sind chronisch-entzündliche Hauterkrankungen, die mit ausgeprägten psychischen Belastungen einhergehen.

\section{Ergebnisse der Studie}

Depressionen werden bei bis zu 42\% der Patienten mit Hs berichtet. Bei Ps wurden in einer Arbeit von Esposito et al. $(n=2391)$ bei $62 \%$ der Patienten depressive Symptome festgestellt; interessanterweise korrelierte in einer weiteren Arbeit der «Psoriasis Area and Severity Index»(PASI)-Wert mit dem Schweregrad der Depression [1]. 31\% der Patienten mit AD in den USA berichteten über mindestens ein depressives Symptom [2]. Auch beobachtet man eine Verschlechterung der dermatologischen Erkrankungen bei psychologischem Stress. Ist diese Assoziation nur durch die negativen psychologischen Effekte der Hauterkrankung wie soziale Ängste und Scham erklärbar oder gibt es einen zugrunde liegenden pathophysiologischen Mechanismus [1]? Inzwischen gilt es als gesichert, dass jede der aufgeführten Erkrankungen eine bestimmte Zytokinsignatur aufweist. Bei der Ps dominiert die Interleukin (IL)-17-Achse mit Beteiligung von IL-12/23 und Tumornekrosefaktor (TNF)-a. Die AD weist eine Dominanz der von T-Helfer-2-Zellen produzierten Zytokinen auf. Bei der Hs scheint eine Dysregulation von TNF-a, IL-1, IL-12/23, IL-17 und IL-6 vorzuliegen. Es gibt viele Daten, die zeigen, dass erhöhte Zytokinspiegel einen psychologischen Einfluss haben können. Zytokine wie IL-6 und TNF-a können im Serum während einer schweren Depression gefunden werden und korrelieren mit deren Schweregrad.

Es wurde gezeigt, dass eine Aktivierung des Immunsystems Veränderungen der Stimmung, wie z.B. das Auftreten von Symptomen einer Depression wie Anhedonie, Anorexie und sozialem Rückzug, bewirken kann.

Auch wenn die Entzündung lokal und in peripheren Organen auftritt, können proinflammatorische Zytokine wie TNF-a und IL-6, aber auch dendritische Zellen die Blut-Hirn-Schranke überwinden. Erhöhte Spiegel beispielsweise an TNF-a können den Abbau von Neurotransmittern wie Serotonin durch Aktivierung von Enzymen wie Indolamin-2,3-Dioxygenase (IDO) beschleunigen. Außerdem ergaben klinische Therapiestudien eine signifikante Reduktion depressiver Symptome mit Zytokininhibitoren wie z.B. Etanercept und Ustekinumab bei Ps, Dupilumab bei AD und Adalimumab bei Hs. Interessanterweise zeigten auch Studien mit Zytokininhibitoren zur Behandlung von Depressionen bei nicht-dermatologischen $\mathrm{Pa}$ tienten ähnliche Ergebnisse.

\section{Wie erkennen wir psychische Erkrankungen?}

Dermatologen sind im beruflichen Alltag regelmäßig mit psychischen Erkrankungen konfrontiert. Die britische Vereinigung der Psychodermatologen schätzt, dass 17\% der dermatologischen Patienten neben der Hauterkrankung psychologische Probleme haben [3]. In einer Arbeit von Dalgard et al. [3] wurden «Hamilton anxiety and depression scale»(HADS)-Fragebogen in dermatologischen Ambulanzen in 13 Ländern verteilt und die Ergebnisse mit den gestellten Diagnosen der Dermatologen verglichen. In einer Analyse von
3635 Ambulanzbesuchen lag die richtig positiv erkannte Rate (d.h. der Prozentsatz der von Dermatologen erkannten Symptome von Depression oder Angststörung bei Patienten mit Depressionen bzw. Angststörungen definiert als HADS-Wert $\geq 11$ ) bei $44 \%$ für Depressionen und 35,6\% für Angststörungen. Psychische Störungen wurden im Praxisalltag unterdiagnostiziert. Ähnlich niedrige Raten zeigten sich auch bei Untersuchungen von Allgemeinmedizinern und Onkologen [3]. Eine Möglichkeit dies zu verbessern, besteht in der Verwendung von speziellen Fragebogen. Eine Arbeitsgruppe der Nationalen Versorgungskonferenz Psoriasis schlägt den «2-Fragen-Test» für das Erkennen von depressiven Symptomen vor: Frage 1: Haben Sie sich im letzten Monat häufig niedergeschlagen, traurig, deprimiert oder hoffnungslos gefühlt?

Frage 2: Haben Sie im letzten Monat deutlich weniger Vergnügen und Freude an Dingen gehabt, die Sie sonst gerne machen?

Wenn beide Fragen mit Ja beantwortet werden, sollte eine Überweisung zum Hausarzt, Psychiater oder Psychotherapeuten erfolgen [4]. Eine weitere Möglichkeit besteht in der Verwendung des Patientengesundheits-Fragebogens (Patient-Health-Questionnaire, PHQ) [5]. Bezogen auf Depressionen kann der PHQ-D2 bzw. PHQ-D9 (2-Fragen- bzw. 9-Fragen-Test) verwendet werden. Er kann ohne Gebühren für nicht-kommerzielle Zwecke genutzt werden.

\section{Fazit für die Praxis}

Die Assoziation von chronischen Hauterkrankungen und psychischen Störungen wie Depressionen sind häufig und nicht einfach zu diagnostizieren. Um diese auch in der täglichen Routine zu erfassen, können der «2-Fragen-Test» oder Fragebögen wie der PHQD2 oder PHQ-D9 eingesetzt werden.

\section{Disclosure Statement}

Hiermit erkläre ich, dass keine Interessenskonflikte in Bezug auf den vorliegenden Wissenstransfer bestehen.

\section{Erstveröffentlichung}

Dieser Beitrag wurde erstveröffentlicht in: Karger Kompass Dermatol 2019;7:16-17.

\section{Literatur}

1 Tohid H, Aleem D, Jackson C: Major depression and psoriasis: a psychodermatological phenomenon. Skin Pharmacol Physiol 2016;29:220-230. Erratum in: Skin Pharmacol Physiol 2016;29:280

2 Nicholas MN, Gooderham MJ: Atopic dermatitis, depression, and suicidality. J Cutan Med Surg 2017;21:237-242.

3 Dalgard FJ, Svensson A, Gieler U, et al.: Dermatologists across Europe underestimate depression and anxiety: results from 3635 dermatological consultations. Br J Dermatol. 2018;179:464-470.

4 Radtke MA, Mrowietz U, Feuerhahn J, et al.: Early detection of comorbidity in psoriasis: recommendations of the national conference on healthcare in psoriasis. J Dtsch Dermatol Ges 2015;13:674-690.

5 Pettersson A, Boström KB, Gustavsson P, et al.: Which instruments to support diagnosis of depression have sufficient accuracy? A systematic review. Nord J Psychiatry 2015;69:497-508.

Kontaktadresse: Dr. Sandra Philipp,Hautarztpraxis Dr. M. Friedrich/ Dr. S. Philipp,Bernauerstraße 66,16515 Oranienburg,

Deutschland,hautarzt.philipp@gmail.com 